\title{
Intratumor genetic heterogeneity in squamous cell carcinoma of the oral cavity
}

\author{
Dan P. Zandberg MD ${ }^{1}$ (c) । Luke J. Tallon $\mathrm{PhD}^{2}$ । Sushma Nagaraj MS ${ }^{2}$ । \\ Lisa K. Sadzewicz $\mathrm{PhD}^{2}$ । Yuji Zhang $\mathrm{PhD}^{3}$ | Maxwell B. Strome ${ }^{4}$ | Xuechu E. Zhao $\mathrm{PhD}^{2}$ । \\ Kranthi Vavikolanu $\mathrm{MS}^{2}$ । Xiaoyu Zhang $\mathrm{PhD}^{5}$ । John C. Papadimitriou MD, $\mathrm{PhD}^{6}$ । \\ Fleesie A. Hubbard MS ${ }^{5}$ ， Søren M. Bentzen $\mathrm{PhD}^{3}$ | Scott E. Strome MD ${ }^{5}$ । \\ Claire M. Fraser $\mathrm{PhD}^{2}$
}

${ }^{1}$ Department of Hematology/Oncology, UPMC Hillman Cancer Center, Pittsburgh, Pennsylvania

${ }^{2}$ Department of Medicine, Institute for Genome Sciences, University of Maryland School of Medicine, Baltimore, Maryland

${ }^{3}$ Department of Epidemiology and Public Health, University of Maryland School of Medicine, Baltimore, Maryland

${ }^{4}$ College of Literature, Science, and the Arts, University of Michigan, Ann Arbor, Michigan

${ }^{5}$ Department of Otorhinolaryngology-Head and Neck Surgery, University of Maryland School of Medicine, Baltimore, Maryland

${ }^{6}$ Department of Pathology, University of Maryland School of Medicine, Baltimore, Maryland

\section{Correspondence}

Dan P. Zandberg, Department of

Hematology/Oncology, UPMC Hillman

Cancer Center, 5150 Centre Avenue, 5th

Floor, Pittsburgh, PA 15232.

Email: zandbergdp@upmc.edu

\begin{abstract}
Background: We sought to evaluate intratumor heterogeneity in squamous cell carcinoma of the oral cavity (OCC) and specifically determine the effect of physical separation and histologic differentiation within the same tumor.

Methods: We performed whole exome sequencing on five biopsy sites-two from well-differentiated, two from poorly differentiated regions, and one from normal parenchyma-from five primary OCC specimens.

Results: We found high levels of intratumor heterogeneity and, in four primary tumors, identified only 0 to 2 identical mutations in all subsites. We found that the heterogeneity inversely correlated with physical separation and that pairs of well-differentiated samples were more similar to each other than analogous poorly differentiated specimens. Only TP53 mutations, but not other purported "driver mutations" in head and neck squamous cell carcinoma, were found in multiple biopsy sites.

Conclusion: These data highlight the challenges to characterization of the mutational landscape of OCC with single site biopsy and have implications for personalized medicine.
\end{abstract}

\section{K E Y W O R D S}

genetic, heterogeneity, SCCHN, oral cavity, squamous cell carcinoma

\section{1 | INTRODUCTION}

Squamous cell carcinoma of the oral cavity (OCC) is a worldwide health problem with an incidence in males of 6.6/100 000 in the United States and 16.4/100 000 in India. ${ }^{1}$ Unlike human papilloma virus (HPV)-associated oropharyngeal carcinomas, ${ }^{2}$ which have exceptionally good outcomes with current treatment modalities, ${ }^{3}$ survival for patients with advanced OCC remains poor. ${ }^{4}$
Improved outcomes for patients afflicted with OCC will likely be predicated on a detailed understanding of the genetic changes that drive tumor proliferation/survival and the correlation of these changes with routine histopathologic findings that are the backbone of current staging systems. For instance, understanding the variability in mutations among select OCC subsites is an important consideration for molecular staging systems, such as the Mutant Allele Tumor Heterogeneity score, which are poised for integration into clinical care. ${ }^{5-7}$ In 
addition, insight into the mutational heterogeneity of OCC is critical to the development of personalized treatment strategies for patients afflicted with this disease. For example, the National Cancer Institute's Molecular Analysis for Therapy Choice (NCI-MATCH) trial relies upon the whole exome and mRNA analysis of a single biopsy sample from a patient's tumor to determine if that individual might be successfully treated with an approved drug. Similarly, based on the recent insight that responses to PD-1:PD-L1 axis blockade are not only dependent on a high mutational burden, ${ }^{8-11}$ but also, upon conservation of specific mutations throughout the tumor, ${ }^{12}$ understanding of mutational heterogeneity may provide a useful adjunct to prospective immunotherapy trials. Finally, knowledge of the distinct mutations that encode for putative neoantigens shared among all and/or the majority of tumor subsites is a prerequisite for the development of neoantigen-specific cancer vaccines. ${ }^{13}$

Initial large collaborative whole exome sequencing (WES) efforts of squamous cell carcinomas of the head and neck (SCCHN) revealed extensive intertumor heterogeneity, with the involved genes generally conserved in less than $10 \%$ of specimens-notable exceptions include TP53, CDKN2A, FAT1, NOTCH1, SYNE1, and MLL2. ${ }^{14,15}$ Subsequent analysis of data in The Cancer Genome Atlas validated and extended these initial observations, confirming the transcription of these genes harboring mutations in $86 \%$ of cases. ${ }^{16,17}$ Unfortunately, with few exceptions, the published studies that define the somatic mutations in OCC and SCCHN do not speak to the issues of intratumor heterogeneity nor do they correlate mutational burden with tumor grade. Given that other tumor types such as renal cell carcinoma and pancreatic cancer demonstrate profound intratumor heterogeneity in both the primary tumor and regional lymph node metastasis, this information is likely critical to our understanding of disease pathogenesis. ${ }^{18-22}$

In this report, we utilized WES to explore the intertumor and intratumor mutational landscape of primary OCC. We describe the development and validation of a method that considers three parameters to assess the probability that a non-synonymous mutation in an OCC exon is valid: (a) identification of a DNA sequence variant with two mutation caller systems, (b) the extent of DNA sequence coverage for each variant, and (c) the normalized allele frequency. Using this approach, we mapped the exomes of four unique sites-two from well and two from poorly differentiated regions-from five primary OCCs.

\section{2 | MATERIALS AND METHODS}

\section{1 | Pathology}

Representative sections (2-3 mm in thickness; at least one section/cm of tumor) were obtained from five randomly selected patients who had undergone surgical resection of their OCC. Sections were fixed in $10 \%$ formaldehyde, processed, and embedded in paraffin blocks, from which $5 \mu \mathrm{m}$ sections were cut and stained with hematoxylin-eosin (H\&E) satin. Selected areas were categorized as "well-differentiated" or "poorly differentiated" by a head and neck pathologist. We selected two areas of well-differentiated (eg, W1 and W2) and two areas of poorly differentiated carcinoma (eg, P1 and P2), as well as one area of normal mucosa $(\mathrm{N})$ from each primary tumor. These areas were circled on the H\&E stained slides and the distances between all of these selected areas were recorded (Figure 1).
FIG URE 1 Approach for individual sample identification. A, B, D, We harvested two areas of poorly differentiated tumor $(\mathrm{P})$ and two areas of welldifferentiated tumor along with histologically normal tissue from the same site (N). For every core biopsy of tumor obtained, a 5- $\mu$ m thick section was cut from this core (biopsy of the biopsy) and the resulting slides were stained with hematoxylin-eosin stain to evaluate purity of differentiation in vertical direction of the sample. C, Distances between samples were mapped in two dimensions for statistical analysis [Color figure can be viewed at wileyonlinelibrary.com]
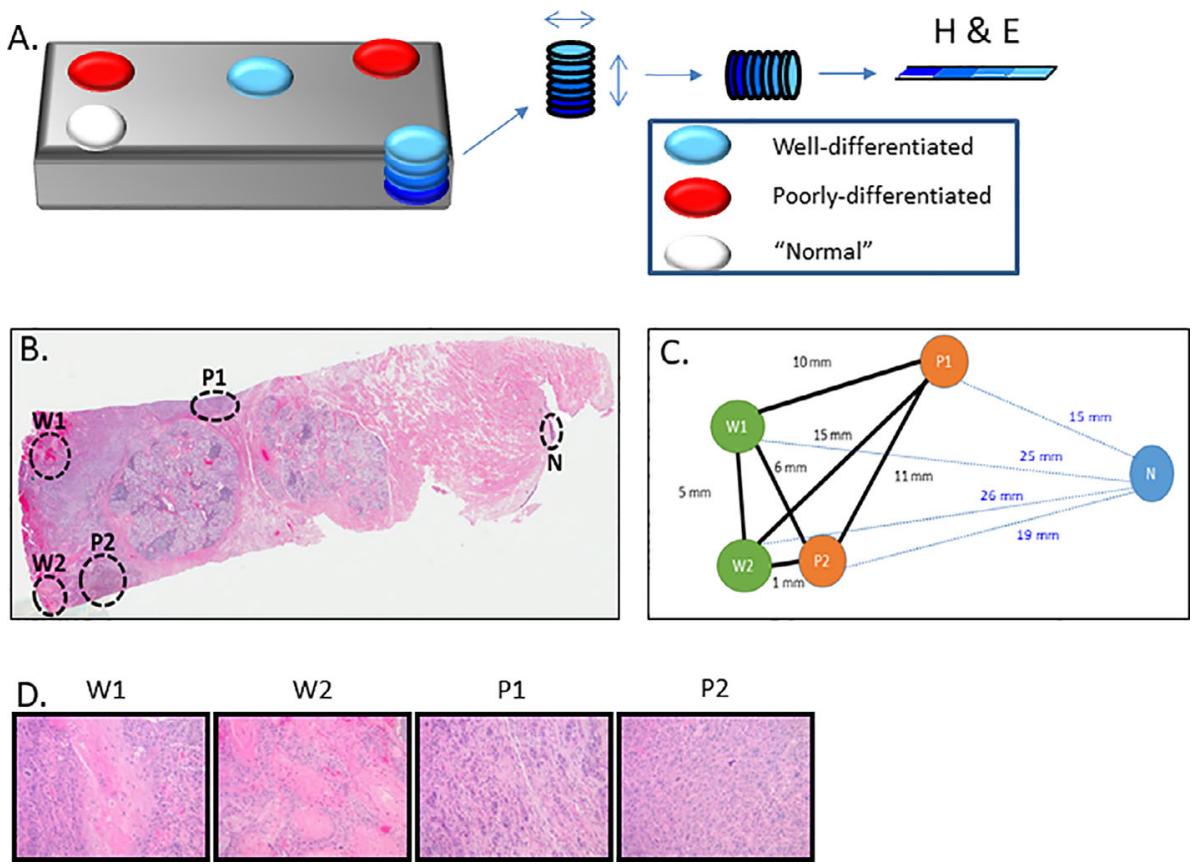
For each of the selected areas, a tissue core $(2.0 \times$ $2.0 \times 2.5 \mathrm{~mm}$ ) was obtained with a metallic biopsy needle from the corresponding paraffin block and re-embedded in a new paraffin block. A $5 \mu \mathrm{m}$ thick section was cut from this new block in the vertical plane and embedded/sectioned in the horizontal plane. The resulting slides were stained with H\&E and used to validate the assigned tumor differentiation category, and to determine the tumor purity throughout the core (ie, \% of tumor/core tissue, "biopsy of the biopsy") (Figure 1 and Supporting Information Table S1). The remaining tissue, which represented most (ie, >90\%) of the punched-out core, was submitted for molecular studies. In addition to histologic evaluation, we employed the recently described informatics tool, Sequenza, as an additional means to evaluate tumor purity. ${ }^{23}$

\section{2 | Fresh frozen and FFPE DNA extractions}

Genomic DNA was extracted from Formalin-Fixed ParaffinEmbedded (FFPE) sections and biopsy cores using Covaris truXTRAC FFPD DNA Kit (Covaris, Woburn, Massachusetts). In brief, FFPE sections less than $25 \mu \mathrm{m}$ thick or biopsy cores smaller than $1.2 \mathrm{~mm}$ in diameter were loaded into Covaris microTUBEs, supplemented with buffer and processed in Covaris E210 instrument to dissociate the paraffin and rehydrate the tissue. Tissue was then lysed with Protease $\mathrm{K}$ overnight at $56^{\circ} \mathrm{C}$ followed by a 1-hour incubation at $80^{\circ} \mathrm{C}$ to reverse formaldehyde crosslinks. Genomic DNA was extracted through a spin column provided in the kit, and further purified with AMPure XT beads (Beckman Coulter Genomics, Danvers, Massachusetts).

\section{3 | Library construction}

Genomic DNA libraries were constructed for sequencing on the Illumina platform using the KAPA Library Preparation Kit (Kapa Biosystems, Wilmington, Massachusetts). First, DNA was fragmented with the Covaris E210. Then, libraries were prepared using a modified version of manufacturer's protocol. The DNA was purified between enzymatic reactions and the size selection of the library was performed with AMPure XT beads (Beckman Coulter Genomics). The polymerase chain reaction (PCR) amplification step was performed with primers containing an index sequence $7 \mathrm{nt}$ in length.

\subsection{Exome capture and sequencing}

Exome capture was performed using the Agilent SureSelect Human All Exon v4 kit (Agilent Technologies, Santa Clara, CA) according to manufacturer's protocol. Multiplexed sequencing was performed on the Illumina HiSeq 4000 platform using (Illumina, San Diego, CA) 151 bp paired-end runs. An average of 518 million reads was generated for each sample.

\section{5 | Data preprocessing and alignment}

Illumina reads were aligned to human reference $\mathrm{v} 37$ with decoy sequences using BWA $^{24}$ version 0.7.12-r1039. Picard ${ }^{25}$ tools (version 1.140) CleanSam, FixMateInformation, and MarkDuplicates were used to clean up any mapping artifacts and mark duplicate read pairs. Insertion/deletion (INDEL) realignment and base quality recalibration were performed using GATK $^{26}$ version 3.3-0. All the tumor and normal samples derived from the same patient were co-realigned. The resulting alignments had an average on-target depth of 284 reads. The median on-target depth was 260x with a range of 142x to 479x. On average, $>99 \%$ of the targeted bases were covered at 30x or more with a range of $96 \%$ to $100 \%$ (Supporting Information Table S2).

\section{6 | Variant calling, annotation, and filtering}

Somatic single-nucleotide variant (SNVs) were called using MuTect ${ }^{27}$ version 1.1.7 and VarScan ${ }^{28}$ version 2.4.1 to obtain a set of high confidence variants. Independent variant calling was done for each sample analyzed. MuTect applies several variant filters to detect any false positive SNVs generated due to mapping and sequencing errors. A variant was identified as a false positive if: (a) it was observed in the matched normal sample, (b) there was a proximal gap, (c) a site exhibited poor mapping or strand bias, (d) alternate alleles were clustered at a consistent distance from the read ends, or (e) the site was triallelic. The VarScan call set was also processed to obtain a high confidence set. Default allele frequency thresholds were applied to variants classified as somatic. If the variant allele frequency was $>5 \%$ in tumor and $<5 \%$ in normal, it was marked as high confidence. Furthermore, variants were filtered to identify false positives due to poor mapping, strand bias, read position bias, etc.

The resulting somatic variants were annotated for functional effect using ANNOVAR ${ }^{29}$ (version 2015-06-17). ANNOVAR was also used to annotate the variants with allele frequencies in the 1000 Genomes Project, NHLBI-ESP 6500 exomes, CLINVAR, COSMIC, NCI60 and to calculate scores for prediction algorithms including, SIFT, PolyPhen, and MutationAssessor. The 1000 Genomes Project alternate allele frequency threshold was set to 0.01 to filter out common variants.

To evaluate the purity of the normal samples, we calculated the allele frequency distribution of each sample. An average of $83 \%$ of variant Allele Frequency (AF) were heterozygous $(0.4 \leq \mathrm{AF} \leq 0.6)$ or homozygous ( $\mathrm{AF}>0.9)$, with a range of $77 \%$ to $86 \%$ (Supporting Information Table S3 and Figure S1).

Because FFPE data carry a risk of artifactual mutations due to cytosine deamination, we evaluated the prevalence of $\mathrm{C}->\mathrm{T}$ and G->A mutations in each sample (Supporting Information Figure S2). Although a number of samples showed significant enrichment for these mutation types, after removing all $\mathrm{C}->\mathrm{T}$ 
and G->A mutations from the final call set, the rate of overlap among the samples changed by less than $1 \%$ (Supporting Information Figure S3 and Table S4).

\section{7 | Somatic variant validation}

All non-synonymous stop loss and stop gain variants that were called with high confidence by both callers were used to pick a set of variants for validation. These were prioritized using MutationAssessor ${ }^{30}$ predictions and cross-referenced with genes known to be associated with cancers (specifically head and neck cancers) using COSMIC ${ }^{31}$ and tumorportal.org, ${ }^{32}$ and a final validation set was chosen. PCR primers for each variant were selected using a combination of Primer3 and manual primer design (Validation PCR primers synthesized by SigmaAldrich, St. Louis, MO). Resulting PCRs were sequenced using barcoded multiplexed runs on a PacBio RS II with P6-C4 chemistry (Pacific Biosciences, Menlo Park, CA). The resulting PacBio CCS reads were aligned to the human reference with BLASR and the alignments and consensus calls were manually inspected using Integrated Genomics Viewer (IGV).

\subsection{Statistical analysis}

The Jaccard similarity coefficient $J(A, B)$ was used for quantifying the co-occurrence of SNVs in two samples from the same patient, $\mathrm{A}$ and $\mathrm{B}$ :

$$
J(A, B)=\frac{|A \cap B|}{|A \cup B|}=\frac{|A \cap B|}{|A|+|B|-|A \cap B|}
$$

For constructing dendrograms, the Jaccard distance or Jaccard dissimilarity defined as $1-J(A, B)$ was used as the distance metric between samples. Dendrograms were produced using Wolfram Mathematica version 11.0.0. A forest plot of the fraction of shared SNVs in individual samples was generated using the "meta" library in the R software. ${ }^{33}$ Partial correlation was used to test the significance of the association between increasing Jaccard similarity and decreasing physical distance between samples with the effect of parent tumor removed. Likewise, the unidirectional hypothesis that Jaccard similarity increases with decreasing physical distance between paired samples in individual tumors was tested using the nonparametric Spearman's rank correlation coefficient, $R_{\mathrm{s}}$. The corresponding $P$ value is for rejecting the hypothesis $R_{\mathrm{s}}=0$. These analyses were conducted using IBM SPSS Statistics version 24.0.0.

\subsection{Study approval}

All studies were conducted under the auspices of a protocol approved by the Institutional Review Board, University of Maryland, Baltimore. As determined by the IRB, the protocol did not require written informed consent.

\section{3 | RESULTS}

\section{1 | Tumor site and stage}

Four study participants had SCC of the oral tongue (patients $5280,1646,6803$, and 7157), whereas one tumor originated in the lower gingiva (9464). Patient's ages ranged from 49 to 89 years. Tumors were pathologically staged by TNM classification as: T2N0, T1N2c, T3N2c, T2N0, and T1N0. None of the patients had distant metastatic disease at time of resection.

\section{2 | Validation of somatic mutations correlates with both allele frequency and sequence coverage}

We analyzed our WES data using two widely employed caller systems, MuTect and VarScan, and only validated mutations called by both systems (Table 1). ${ }^{27,28}$ We performed 190 PCR reactions for overlapping SNVs and successfully validated $139(73 \%)$. The frequency of allele detection and sequence coverage directly correlated with successful validation (Table 1 , Figure 2). With sequence coverage greater than 500 , the validation rate was $79 \%$ and for allele frequency greater than 0.5 , the validation rate was $86 \%$. Combining both of these criteria resulted in a validation rate of $100 \%$. Lowering the coverage to $>250$ with allele frequency $>0.5$ still produced a $92 \%$ validation rate. Collectively, these data define the likelihood that a mutation identified by our approach in silico is valid based on set parameters.

T A B L E 1 Validation by allele frequency and coverage

\begin{tabular}{lllllll} 
Allele frequency & \multicolumn{2}{l}{ All validation attempts } & \multicolumn{3}{l}{ PCR+ validation attempts } \\
\hline$>0.75$ & $5 / 11(45 \%)$ & $3 / 5(60 \%)$ & $1 / 1(100 \%)$ & $5 / 6(83 \%)$ & $3 / 4(75 \%)$ & $1 / 1(100 \%)$ \\
$0.5-0.75$ & $14 / 19(74 \%)$ & $9 / 10(90 \%)$ & $11 / 11(100 \%)$ & $14 / 18(78 \%)$ & $9 / 10(90 \%)$ & $11 / 11(100 \%)$ \\
\hline $0.25-0.5$ & $17 / 38(45 \%)$ & $17 / 32(53 \%)$ & $14 / 25(56 \%)$ & $17 / 30(57 \%)$ & $17 / 26(65 \%)$ & $14 / 21(67 \%)$ \\
$<0.25$ & $11 / 36(31 \%)$ & $11 / 26(42 \%)$ & $16 / 24(67 \%)$ & $11 / 25(44 \%)$ & $11 / 18(61 \%)$ & $16 / 20(80 \%)$ \\
\hline Coverage & $0-250$ & $250-500$ & $>500$ & $0-250$ & $250-500$ & $>500$
\end{tabular}

"All validation attempts" refers to all validation attempts, including polymerase chain reaction (PCR) failures. The "PCR+ validation attempts" set includes only those validation attempts where a PCR amplicon was obtained. 


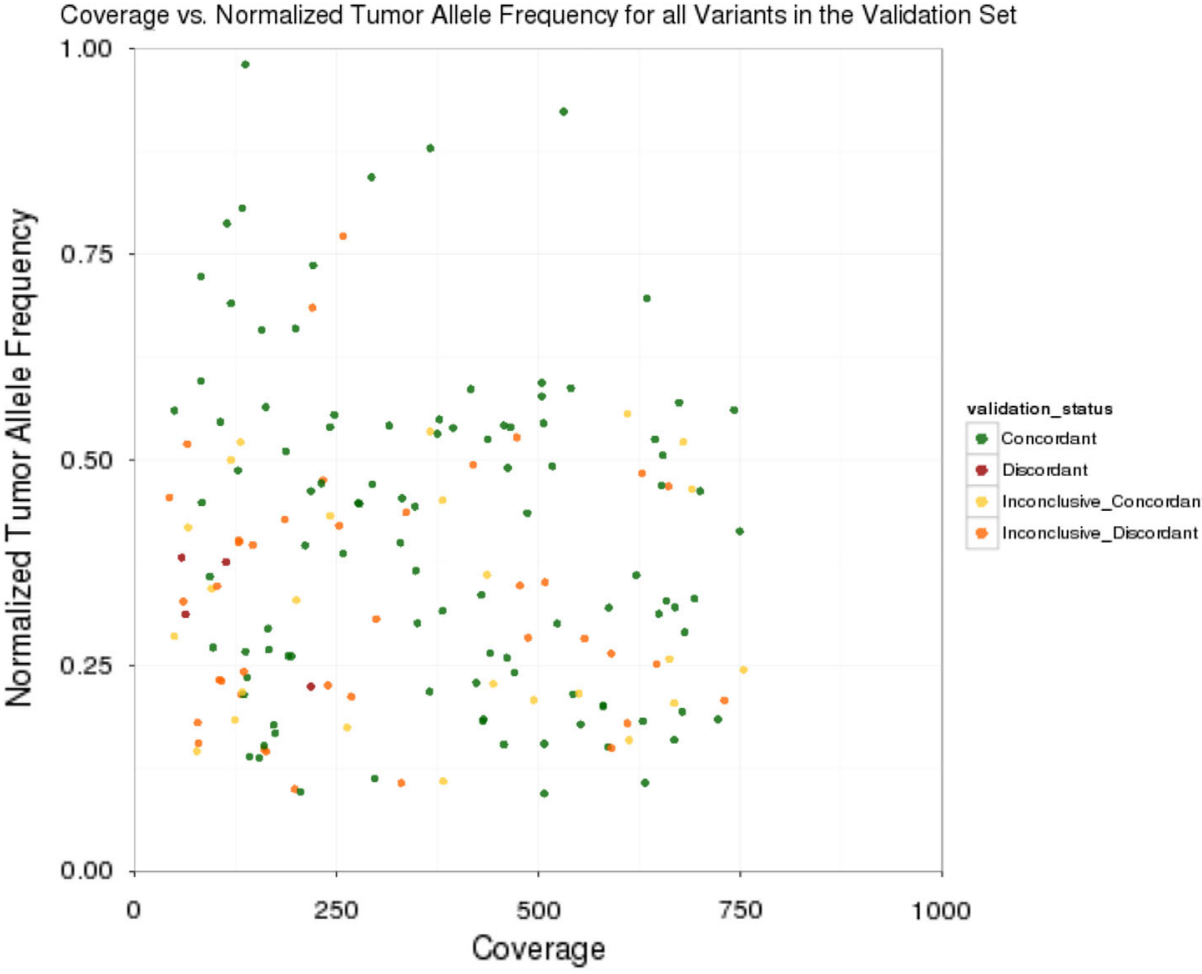

FIGURE 2 Mutation validation as a factor of coverage and allele frequency. Selected mutations were validated by polymerase chain reaction (PCR) and PacBio sequencing from a range of coverages and allele frequencies. Tumor allele frequencies were normalized by expected tumor purity values. Validation attempts with strong high-coverage support are labeled concordant or discordant. Those with low-coverage support are labeled inconclusive concordant or discordant [Color figure can be viewed at wileyonlinelibrary.com]

\section{3 | Primary OCCs have variable mutational burdens}

In order to determine if the mutational burden of OCC was consistent throughout primary OCCs, we evaluated high impact SNVs - defined as non-synonymous or stop mutations-in four samples from each primary tumor (Table 2). We identified 635 SNVs, with each primary tumor having variable tumor wide and subsite specific mutational burdens (range 60-223 per patient tumor) (Figure 3A). For example, in tumors 5280 and 1646, two of the subsites had high and two had low mutational burden indices. In contrast, two tumors (9464 and 6803) displayed high mutational burdens with internal consistency. Finally, the mutational burden index was approximately equal in three of the four subsites from tumor 7157. These data demonstrate that OCCs have variable levels of intratumor and intertumor mutational burdens that contribute to their overall genetic diversity.

\section{4 | Primary OCCs have high level of intratumor heterogeneity}

In general, these tumors had high levels of mutation and gene-specific intratumor heterogeneity. For example, in four primary tumors, we identified only zero to two identical mutations in all subsites-a finding that correlated with a $0.7 \%$ median SNV overlap per patient. For the three patients that did share at least one common SNV in all four sub sites, each SNV corresponded to one gene (Supporting Information

T A B L E 2 Comparison of overlap of SNV's in tumor samples from all patients

\begin{tabular}{|c|c|c|c|c|c|c|c|c|c|c|c|}
\hline Patient & Site stage ${ }^{a}$ & W1 & W2 & $\begin{array}{l}\text { Distance } \\
(\mathbf{m m})^{\mathbf{b}}\end{array}$ & $\begin{array}{l}\text { Shared } \\
\text { SNVs }^{c} n(\%)\end{array}$ & $\mathbf{P} 1^{\mathbf{d}}$ & $\mathbf{P 2}$ & $\begin{array}{l}\text { Distance } \\
(\mathbf{m m})^{\mathbf{b}}\end{array}$ & $\begin{array}{l}\text { Shared } \\
\text { SNVs }^{\mathrm{c}} \mathrm{n}(\%)\end{array}$ & $\begin{array}{l}\text { Total unique } \\
\text { SNVs }\end{array}$ & $\begin{array}{l}\text { Total shared } \\
\text { SNVs } \\
\text { n(\%) }\end{array}$ \\
\hline 5280 & Tongue $\mathrm{T} 2 \mathrm{~N} 0$ & 29 & 81 & 2.5 & $22(25)$ & 15 & 59 & 0.5 & $1(1)$ & 149 & $1(0.7)$ \\
\hline 1646 & Tongue T3N2c & 58 & 68 & 5 & $56(80)$ & 5 & 12 & 11 & $0(0)$ & 81 & $0(0)$ \\
\hline 9464 & Gingiva T1N2c & 128 & 126 & 2 & $122(92)$ & 131 & 128 & 1 & 42 (19) & 223 & $41(18)$ \\
\hline 6803 & Tongue $\mathrm{T} 2 \mathrm{~N} 0$ & 57 & 56 & 10 & $55(95)$ & 48 & 0 & 7 & $0(0)$ & 60 & $0(0)$ \\
\hline 7157 & Tongue T1N0 & 5 & 78 & 15 & $2(2)$ & 84 & 93 & 1 & $58(49)$ & 122 & $2(1.6)$ \\
\hline
\end{tabular}

Variants are Non-synonymous exonic SNV's discovered by both calling systems.

${ }^{\text {a }}$ Staging represents pathologic stage.

${ }^{\mathrm{b}}$ Distance between samples (W1-W2 or P1-P2).

${ }^{\mathrm{c}}$ Found in both samples within a patients tumor (W1 and W2 or P1 and P2).

${ }^{\mathrm{d}} \mathrm{W}$, well-differentiated sample; $\mathrm{P}$, poorly differentiated sample. Numeric value represents the total number of SNVs in each sample.

${ }^{\mathrm{e}}$ Total number of unique SNVs in all four samples for each patient.

${ }^{\mathrm{f}}$ Found in all four samples within a patient's tumor. Each SNV corresponds to one gene. 
(A)

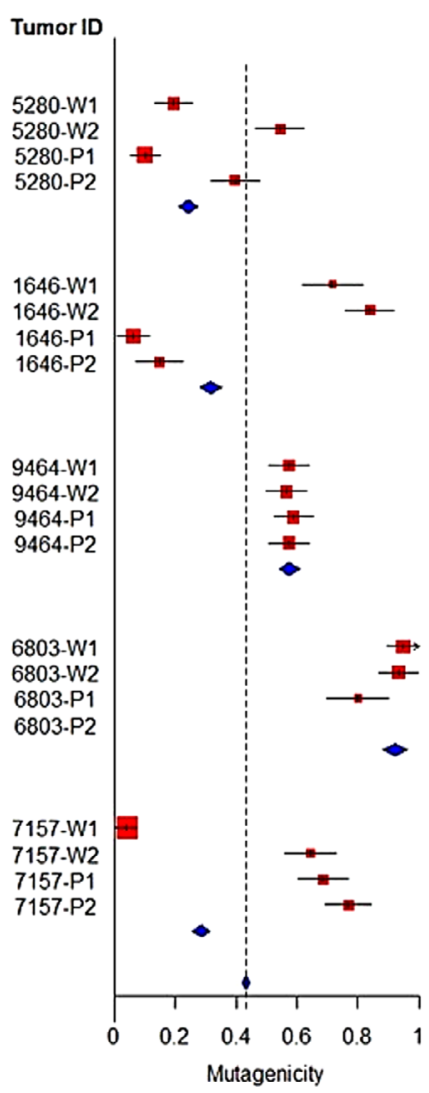

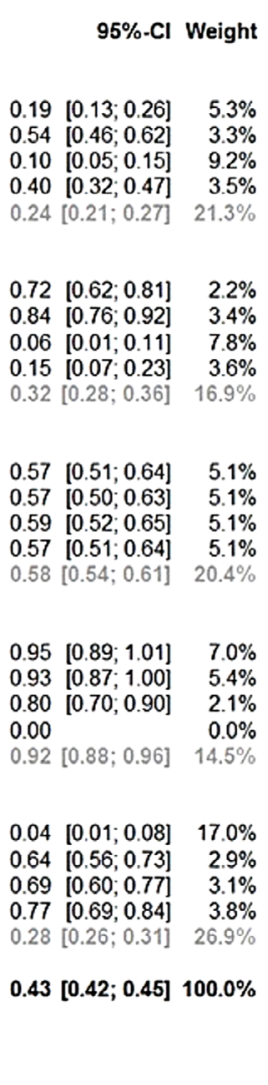

\begin{tabular}{|c|c|c|c|}
\hline Sample name & $\begin{array}{l}\text { Sequenza } \\
\text { Estimated } \\
\text { Tumor } \\
\text { Cellularity }\end{array}$ & $\begin{array}{l}\text { Sequenza } \\
\text { Estimated } \\
\text { Ploidy }\end{array}$ & $\begin{array}{l}\text { Estimated } \\
\text { tumor purity } \\
\text { by pathology }\end{array}$ \\
\hline 5280-W1 & $60 \%$ & 2.1 & $40 \%$ \\
\hline $5280-W 2$ & $24 \%$ & 6 & $100 \%$ \\
\hline 5280-P1 & $58 \%$ & 2.1 & $35 \%$ \\
\hline $5280-\mathrm{P} 2$ & $13 \%$ & 2.3 & $35 \%$ \\
\hline 1646-P1 & $42 \%$ & 2.1 & $20 \%$ \\
\hline 1646-P2 & $49 \%$ & 2 & $70 \%$ \\
\hline $1646-W 1$ & $27 \%$ & 4.1 & $80 \%$ \\
\hline $1646-W 2$ & $62 \%$ & 3.7 & $100 \%$ \\
\hline 9464-W1 & $36 \%$ & 4.1 & $70 \%$ \\
\hline $9464-W 2$ & $69 \%$ & 4.1 & $95 \%$ \\
\hline 9464-P1 & $33 \%$ & 3.6 & $25 \%$ \\
\hline 9464-P2 & $36 \%$ & 4.1 & $65 \%$ \\
\hline 6803-P1 & $35 \%$ & 3.9 & $100 \%$ \\
\hline 6803-P2 & $54 \%$ & 2 & $15 \%$ \\
\hline 6803-W1 & $26 \%$ & 4 & $60 \%$ \\
\hline $6803-W 2$ & $19 \%$ & 4.2 & $85 \%$ \\
\hline 7157-W1 & $61 \%$ & 1.9 & $10 \%$ \\
\hline 7157-W2 & $81 \%$ & 1.9 & $80 \%$ \\
\hline 7157-P1 & $80 \%$ & 1.8 & $85 \%$ \\
\hline 7157-P2 & $34 \%$ & 3.7 & $45 \%$ \\
\hline
\end{tabular}

F I G URE 3 Squamous cell carcinoma of the oral cavity (OCC) has high intratumoral and intertumoral heterogeneity. A, Mutational burden index - the percentage of mutations in a given sample/total number of mutations for a given patient. Blue diamond, average mutational burden for each primary tumor. W, well differentiated; P, poorly differentiated. Table shows tumor purity by Sequenza sequence-based analysis vs pathology assessment. B, Venn diagrams showing the overlap of mutations in shared genes between individual sites. C, Dendrograms created using the Jaccard dissimilarity coefficient between pairs of samples from within each tumor. X-axis is the value of the Jaccard coefficient. The Jaccard dissimilarity coefficient is the measure of mutational distance between pairs of samples. The Jaccard dissimilarity coefficient is the proportion of all somatic mutations present in one of the two samples in a pair that are present in both samples [Color figure can be viewed at wileyonlinelibrary.com]

Table S5). Interestingly, in two of these patients, we found a relatively high percentage of identical mutations, in three of the four subsites. Similarly, in tumor 9464, despite the discovery of 41 identical mutations found in all four subsites, individual regions showed high numbers of mutations that were either unique or shared by only one other area. (Figure 3B, Supporting Information Table S5).

To quantify the mutational variability among samples from within a tumor, we computed dendrograms with the Jaccard dissimilarity coefficient as the measure of mutational distance between pairs of samples (Figure 3C). The Jaccard dissimilarity coefficient is the proportion of all somatic mutations present in one of the two samples in a pair that are present in both samples. These data demonstrate considerable tumorto-tumor variation in the mutational relatedness among samples. As an example, tumor 1646 shows a relatively higher similarity within the poorly differentiated and the welldifferentiated samples than between well vs poorly differentiated samples. The independent sample Kruskal-Wallis test showed that in this small sample, the distribution of the Jaccard coefficient was not statistically significantly different among the tumors $(P=0.19)$.

\subsection{Intratumor heterogeneity is partially constrained by physical distance and the degree of histologic differentiation}

Within each tumor, the relationship between the distance between samples and their mutational profiles was variable. However, combined analysis of four tumors (6803 was not included because P2 had no SNVs called by both calling systems) showed samples that were physically closer together shared more common SNVs $(P=0.03)$. Furthermore, welldifferentiated samples within each tumor had a higher percentage of shared SNVs (median 80\% overlap) compared to poorly differentiated specimens (Median overlap 1.3\%) $(P=0.06)$ (Table 2$)$. This trend was not explained by physical distance between samples as there was no difference in 

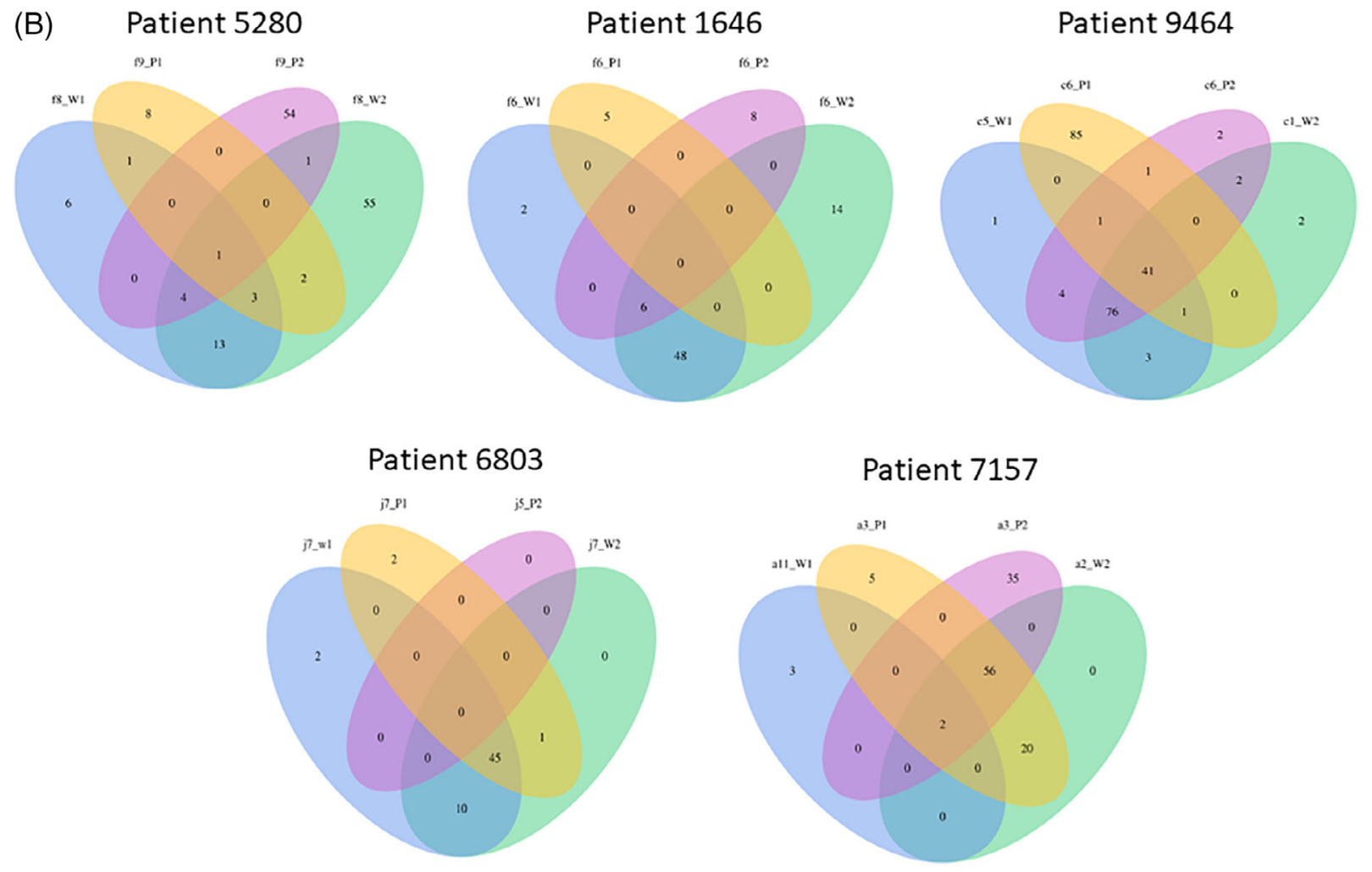

(C) 5280

1646

9464

6803

7157
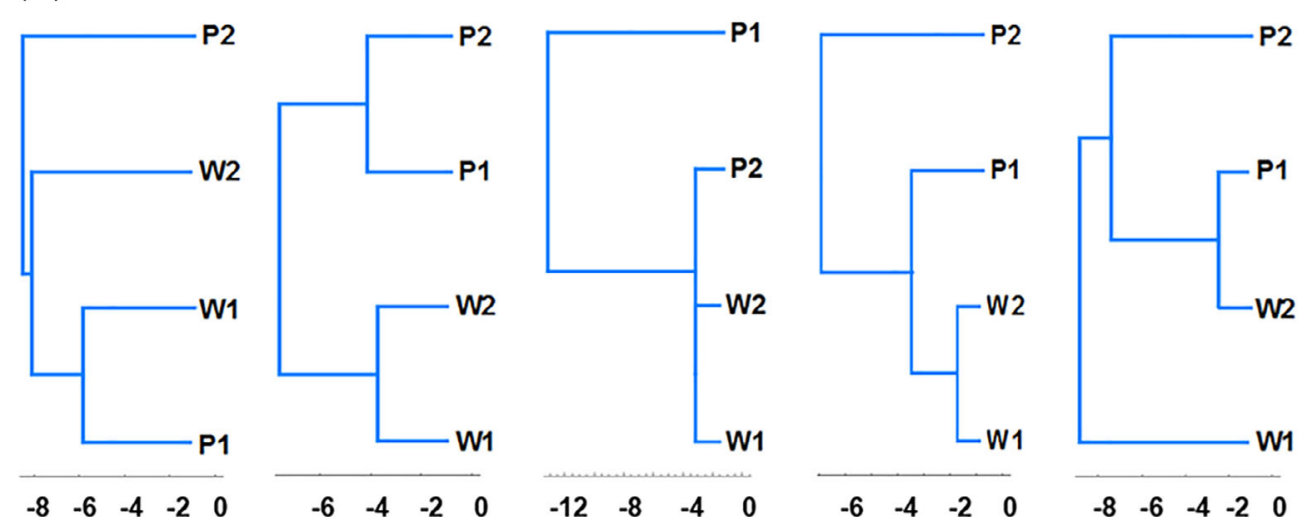

F I G URE 3 (continued) [Color figure can be viewed at wileyonlinelibrary.com]

proximity between the well and poorly differentiated samples $(P=0.1)$. Importantly, as highlighted in Figure $3 \mathrm{C}$, in some tumors (5280 [W1 and P1], 9464 [P2 and W2/W1], and 7157 [P1 and W2]), well-differentiated samples shared more SNVs with poorly differentiated samples, and in all of these cases tumor samples were of short distance from each other (each $3 \mathrm{~mm}$ ) (Supporting Information Table S6).

\subsection{Majority of SNVs identified are in genes not typically associated with SCCHN}

Previous WES studies have identified a group of mutated genes that are conserved in a relatively small number of
SCCHN specimens. ${ }^{14-17}$ Using the two-caller approach, we found only one gene, TP53, which was mutated in more than one patient (Supporting Information Table S5). NOTCH1 and NOTCH2, CASP8, and SYNE1 were detected in two to four of the primary tumor specimens but, generally, not by both callers. Furthermore, unlike TP53 and CDKN2A, in which conserved tumor-specific SNVs were identified by both calling systems in all four subsites throughout an individual tumor (patient 9464), when present, NOTCH1 and NOTCH2, CASP8, and SYNE1 were only found in one to two primary tumor subsites, even when considering calls made by only one calling system. Finally, even with TP53, we did not identify any common SNVs between patients. 


\section{4 | DISCUSSION}

WES of four spatially separated primary tumor samples from each of five patients with OCC demonstrated significant intertumor and intratumor variability in both mutational burden indices and specific somatic SNVs. Interestingly, the physical distance between samples had a significant inverse correlation with their genetic similarity. Furthermore, pairs of welldifferentiated samples trended toward sharing greater numbers of conserved SNVs than their poorly differentiated analogues.

There is limited information on the mutational burden and the mutational heterogeneity of OCC and SCCHN in general. In the most comprehensive study to date, SNV heterogeneity of OCC was analyzed with a 202-gene "cancer gene" panel using two to three distinct samples from each primary tumor and zero to two samples from one lymph node/patient. The findings from this study indicate a high degree of intratumor concordance among the distinct areas sampled from the four patients with oral tongue cancer, with dramatically higher levels of heterogeneity in the one patient with a floor of mouth malignancy. ${ }^{34}$ The differences observed between this report and our study may be as a result of the small sample sizes examined and/or by the use of closed vs open data sets, respectively - the latter is a supposition reinforced by our observation that the majority of SNVs found in our study occurred in genes not commonly associated with SCCHN. Taken in concert, these data suggest that while multiple subsites from individual OCCs share common mutations in the exomes of a defined group of cancer relevant genes, this overlap does not reflect the profound heterogeneity captured by WES. Similar to our study, for example, a high level of intratumor heterogeneity was identified using the whole genome sequencing of an HPV-positive oropharyngeal cancer patient. ${ }^{35}$

Previous WES studies have shown that certain genes are preferentially targeted for mutations in SCCHN ${ }^{14-17}$ Based on these data, there is speculation that some of these genes, for example, TP53 and NOTCH1, are integral to the pathophysiology of these tumors in select patients. We found that TP53 was mutated in a large number of tumor subsites in more than one patient. These findings are consistent with recent studies in non-small cell lung cancer, demonstrating that TP53 driver mutations are generally clonal. ${ }^{21}$ However, while NOTCH1 and NOTCH2, CASP8, and SYNE1 were detected in two to four of the primary tumor specimens, they were only identified in one to two subsites, even when we liberalized our detection approach to the use of a single caller system. While the interpretation of these findings is debatable, they appear to suggest that these genes may not be required in $\mathrm{OCC}$ - at least in certain tumor subsitesand, unlike in squamous cell lung cancer, might have occurred later in the process of tumor progression. ${ }^{21,36}$
In addition to carefully characterizing the mutational heterogeneity of OCC, we asked whether the degree of overlap observed correlated with either the distance between samples and/or histologic differentiation. As anticipated, the distance between samples was inversely correlated with the number of shared SNVs. The fact that these findings were not absolute suggests that OCCs may adhere to both linear as well as nonlinear genetic models of progression. ${ }^{37-39}$ In the long term, it will be important to determine whether, the molecular pathways used for tumor growth and survival in these distinct regions move toward concordance, as is observed in other tumors. ${ }^{40-42}$

To the best of our knowledge, this is the first evaluation of the effect of histologic differentiation on intratumor genetic heterogeneity. Well-differentiated areas within the same patient's tumor trended toward sharing a higher percentage of common SNVs than poorly differentiated regions. This effect was not explained by distance, as well-differentiated samples were not significantly closer to each other than poorly differentiated samples. Also, this observation does not appear to be related to the absolute number of SNVs identified, as both poor and well-differentiated samples varied in mutational burden. Although the small sample size of our study mandates that these data be interpreted with caution, they suggest that mutational heterogeneity might correlate with the degree of histologic differentiation in OCC. Further studies will be required to validate or refute this finding.

There are several limitations to our study. First, because of the relatively small sample size, our observations will need to be validated in a larger patient cohort. Second, while we developed and validated a "two caller" screening algorithm for mutant allele detection that may prove useful for estimating the frequency of "valid calls" in cases where concomitant RNA Seq is not planned, the requirement that both callers identify the same mutation almost certainly decreased the sensitivity of SNV detection and may have led to a higher false negative rate. However, as shown in Supporting Information Figures S4 and S5, the use of one calling system only decreased the intratumor heterogeneity slightly or not at all when comparing all samples. Additionally, as highlighted in Table 3, in only one patient for one gene (TP53 in 5280 in P1 called by MuTect but not VarScan) was there a discrepancy in which the mutation was not found in all samples as a result of using two calling system. We analyzed our validation data set, and found only 10 SNV positions where the validation PCRs detected the variant in a tumor sample where it was not predicted (ie, the variant was present but missed by our algorithm). Of these, four were found by neither MuTect of VarScan, one was found by MuTect but not VarScan, and five were found by MuTect but rejected as poor-quality or likely contamination. Therefore, we do not feel that the use of a two-caller screening algorithm significantly affected our results. 
T A B L E 3 Mutations in commonly reported genes in squamous cell carcinomas of the head and neck (SCCHN) by patient and tumor sample

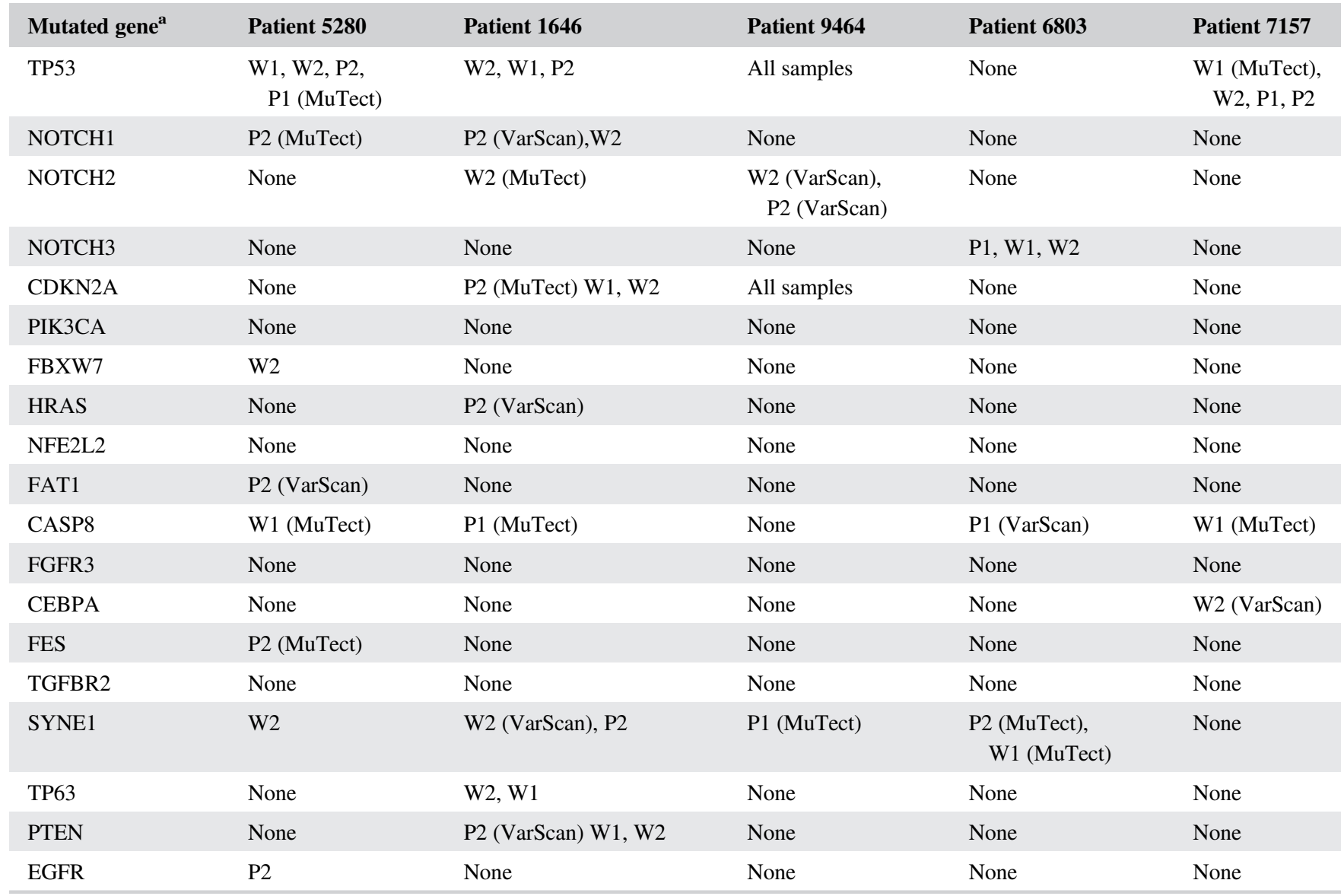

Commonly reported genes, as reported by The Cancer Genome Atlas data.

${ }^{\mathrm{a}}$ Mutation was called by both MuTect and VarScan unless specified.

A third limitation of our study, and all genomics studies comparing samples, is the variability in tumor purity between samples. In order to better understand this inherent variability, tumor purity was defined using both "2-dimensional" H\&E staining and analysis with the recently developed informatics tool, Sequenza. ${ }^{23}$ The fact that these methods had variable concordance highlights the fact that better analytic tools are required to evaluate tumor purity.

Finally, to improve the functional relevance of our study, we only included non-synonymous SNV mutations in our analysis. Because somatic insertion/deletion calling carries a higher error rate, we elected to focus on SNVs to provide a more accurate call set from which to conduct intratumor comparisons. The exclusion of synonymous mutations and INDELs from our analysis reduced estimates of mutational burden, with the effects on mutational heterogeneity being less certain. Finally, the observation in certain patients that large numbers of unique SNVs were found in only one of the four regions analyzed suggests that our study likely under represents the mutational heterogeneity of OCC.

\section{5 | CONCLUSION}

To the best of our knowledge, this is the first report to characterize the mutational burden and mutational heterogeneity of OCC using a WES approach. ${ }^{34,35}$ Our findings indicate that OCCs have variable levels of mutational burden and high levels of intratumor heterogeneity-heterogeneity that is inversely correlated with the physical distance between specimens and is less variable in well vs poorly differentiated regions. Our data also suggest that some of the mutations, for example, NOTCH1, that were postulated to be drivers of tumorigenesis in select patients may not be required in all tumor subsites. Finally, from a therapeutic perspective, our data highlight the challenges of using a single site biopsy for "precision medicine."

\section{ACKNOWLEDGMENTS}

None. 


\section{CONFLICT OF INTEREST}

Dr Strome is a cofounder, consultant, and stockholder in Gliknik Inc., a biotechnology company. He receives royalties for intellectual property, related to B7-H1 (PD-L1), licensed by the Mayo Clinic College of Medicine to third parties. He receives research support from Pfizer and Gliknik through sponsored research agreements through the University of Maryland, Baltimore. He also has served on advisory boards to Astra Zeneca and Genentech. Dr Zandberg receives research support for his role a principal investigator for clinical trials from Merck, Astrazeneca, MedImmune, and Bristol Myers Squibb. All other authors have no relevant disclosures.

\section{ORCID}

\section{Dan P. Zandberg (1) https://orcid.org/0000-0002-1002-8301}

\section{REFERENCES}

1. Chaturvedi AK, Anderson WF, Lortet-Tieulent J, et al. Worldwide trends in incidence rates for oral cavity and oropharyngeal cancers. J Clin Oncol. 2013;31:4550-4559.

2. Strome SE, Savva A, Brissett AE, et al. Squamous cell carcinoma of the tonsils: a molecular analysis of HPV associations. Clin Cancer Res. 2002;8:1093-1100.

3. Ang KK, Harris J, Wheeler R, et al. Human papillomavirus and survival of patients with oropharyngeal cancer. $N$ Engl J Med. 2010;363:24-35.

4. D'Cruz AK, Vaish R, Kapre N, et al. Elective versus therapeutic neck dissection in node-negative oral cancer. N Engl J Med. 2015; 373:521-529.

5. Mroz EA, Tward AD, Hammon RJ, Ren Y, Rocco JW. Intratumor genetic heterogeneity and mortality in head and neck cancer: analysis of data from the Cancer Genome Atlas. PLoS Med. 2015;12:e1001786.

6. Rocco JW. Mutant allele tumor heterogeneity (MATH) and head and neck squamous cell carcinoma. Head Neck Pathol. 2015;9: $1-5$.

7. Mroz EA, Rocco JW. Intra-tumor heterogeneity in head and neck cancer and its clinical implications. World J Otorhinolaryngol Head Neck Surg. 2016;2:60-67.

8. Snyder A, Makarov V, Merghoub T, et al. Genetic basis for clinical response to CTLA-4 blockade in melanoma. $N$ Engl J Med. 2014;371:2189-2199.

9. Rizvi NA, Hellmann MD, Snyder A, et al. Cancer immunology. Mutational landscape determines sensitivity to PD-1 blockade in non-small cell lung cancer. Science. 2015;348:124-128.

10. Le DT, Uram JN, Wang H, et al. PD-1 blockade in tumors with mismatch-repair deficiency. N Engl J Med. 2015;372:2509-2520.

11. Le DT, Durham JN, Smith KN, et al. Mismatch repair deficiency predicts response of solid tumors to PD-1 blockade. Science. 2017; 357:409-413.

12. McGranahan N, Furness AJ, Rosenthal R, et al. Clonal neoantigens elicit $\mathrm{T}$ cell immunoreactivity and sensitivity to immune checkpoint blockade. Science. 2016;351:1463-1469.
13. Melief CJM, van Hall T, Arens R, Ossendorp F, van der Burg SH. Therapeutic cancer vaccines. J Clin Invest. 2015;125:3401-3412.

14. Stransky N, Egloff AM, Tward AD, et al. The mutational landscape of head and neck squamous cell carcinoma. Science. 2011; 333:1157-1160.

15. Agrawal N, Frederick MJ, Pickering CR, et al. Exome sequencing of head and neck squamous cell carcinoma reveals inactivating mutations in NOTCH1. Science. 2011;333:1154-1157.

16. The Cancer Genome Atlas Network. Comprehensive genomic characterization of head and neck squamous cell carcinomas. Nature. 2015;517:576-582.

17. Yan LI, Zhan $\mathrm{C}, \mathrm{Wu}$ J, Wang S. Expression profile analysis of head and neck squamous cell carcinomas using data from The Cancer Genome Atlas. Mol Med Rep. 2016;13:4259-4265.

18. Gerlinger M, Rowan AJ, Horswell S, et al. Intratumor heterogeneity and branched evolution revealed by multiregion sequencing. $N$ Engl J Med. 2012;366:883-892.

19. Yachida S, Jones S, Bozic I, et al. Distant metastasis occurs late during the genetic evolution of pancreatic cancer. Nature. 2010; 467:1114-1117.

20. de Bruin EC, McGranahan N, Mitter R, et al. Spatial and temporal diversity in genomic instability processes defines lung cancer evolution. Science. 2014;346:251-256.

21. Jamal-Hanjani M, Wilson GA, McGranahan N, et al. Tracking the evolution of non-small-cell lung cancer. $N$ Engl J Med. 2017;376: 2109-2121.

22. Zhang J, Fujimoto J, Zhang J, et al. Intratumor heterogeneity in localized lung adenocarcinomas delineated by multiregion sequencing. Science. 2014;346:256-259.

23. Favero F, Joshi T, Marquard AM, et al. Sequenza: allele-specific copy number and mutation profiles from tumor sequencing data. Ann Oncol. 2015;26:64-70.

24. Li H, Durbin R. Fast and accurate short read alignment with Burrows-Wheeler transform. Bioinformatics. 2009;25:1754-1760.

25. Li H, Handsaker B, Wysoker A, et al. The Sequence Alignment/ Map format and SAMtools. Bioinformatics. 2009;25:2078-2079.

26. Van der Auwera GA, Carneiro MO, Hartl C, et al. From FastQ data to high confidence variant calls: the Genome Analysis Toolkit best practices pipeline. Curr Protoc Bioinformatics. 2013;43:11 10-11-33.

27. Cibulskis K, Lawrence MS, Carter SL, et al. Sensitive detection of somatic point mutations in impure and heterogeneous cancer samples. Nat Biotech. 2013;31:213-219.

28. Koboldt DC, Zhang Q, Larson DE, et al. VarScan 2: somatic mutation and copy number alteration discovery in cancer by exome sequencing. Genome Res. 2012;22:568-576.

29. Wang K, Li M, Hakonarson H. ANNOVAR: functional annotation of genetic variants from high-throughput sequencing data. Nucleic Acids Res. 2010;38:e164.

30. Reva B, Antipin Y, Sander C. Predicting the functional impact of protein mutations: application to cancer genomics. Nucleic Acids Res. 2011;39:e118.

31. Forbes SA, Beare D, Gunasekaran P, et al. COSMIC: exploring the world's knowledge of somatic mutations in human cancer. Nucleic Acids Res. 2015;43:D805-D811.

32. Lawrence MS, Stojanov P, Mermel CH, et al. Discovery and saturation analysis of cancer genes across 21 tumour types. Nature. 2014;505:495-501.

33. Schwarzer G. meta: an $\mathrm{R}$ package for meta-analysis. $R$ News. 2007;7:40-45. 
34. Ledgerwood LG, Kumar D, Eterovic AK, et al. The degree of intratumor mutational heterogeneity varies by primary tumor subsite. Oncotarget. 2016;7:27185-27198.

35. Zhang $\mathrm{XC}, \mathrm{Xu} \mathrm{C}$, Mitchell RM, et al. Tumor evolution and intratumor heterogeneity of an oropharyngeal squamous cell carcinoma revealed by whole-genome sequencing. Neoplasia. 2013;15: 1371-1378.

36. McGranahan N, Swanton C. Clonal heterogeneity and tumor evolution: past, present, and the future. Cell. 2017;168:613-628.

37. Califano J, van der Riet P, Westra W, et al. Genetic progression model for head and neck cancer: implications for field cancerization. Cancer Res. 1996;56:2488-2492.

38. Fearon ER, Vogelstein B. A genetic model for colorectal tumorigenesis. Cell. 1990;61:759-767.

39. Nowell P. The clonal evolution of tumor cell populations. Science. 1976;194:23-28.

40. Fisher R, Horswell S, Rowan A, et al. Development of synchronous VHL syndrome tumors reveals contingencies and constraints to tumor evolution. Genome Biol. 2014;15:433.
41. Campbell PJ, Yachida S, Mudie LJ, et al. The patterns and dynamics of genomic instability in metastatic pancreatic cancer. Nature. 2010;467:1109-1113.

42. Gerlinger M, Horswell S, Larkin J, et al. Genomic architecture and evolution of clear cell renal cell carcinomas defined by multiregion sequencing. Nat Genet. 2014;46:225-233.

\section{SUPPORTING INFORMATION}

Additional supporting information may be found online in the Supporting Information section at the end of this article.

How to cite this article: Zandberg DP, Tallon LJ, Nagaraj S, et al. Intratumor genetic heterogeneity in squamous cell carcinoma of the oral cavity. Head \& Neck. 2019;41:2514-2524. https://doi.org/10.1002/ hed.25719 